\title{
Cyclohexenones as Michael Acceptors in the Staunton- Weinreb Annulation: A Simple Stannane Modification for the Synthesis of Polycyclic Systems
}

\author{
Bryan Hill ${ }^{\dagger}$ and Russell Rodrigo* \\ Department of Chemistry, University of Waterloo, 200 University Avenue West, \\ Waterloo, Ontario, Canada N2L 3G1 \\ rrodrigo@uwaterloo.ca
}

\section{Supporting Information}

General Information: Tetrahyrofuran (THF) was dried and distilled over $\mathrm{Na}$ /benzophenone and diisopropylamine was dried and distilled over $\mathrm{CaH}_{2}$. All other reagents were purchased and used as obtained from commercial sources. All glassware was dried in a oven and stored in a dry box prior to use. All NMR spectra were recorded in $\mathrm{CDCl}_{3}$ on a Bruker 300 Avance spectrometer and reported at $\delta 7.24$ and $\delta 77.0$ for the ${ }^{1} \mathrm{H}$ and ${ }^{13} \mathrm{C}$ respectively.<smiles>CCCCCc1cccc(OC)c1C(=O)OCC</smiles>

Stannane 1: To a solution of $i-\mathrm{Pr}_{2} \mathrm{NH}$ (3.24 mL, $\left.23.2 \mathrm{mmole}\right)$ and THF (90 mL) at $0^{\circ} \mathrm{C}$ was added $n$-BuLi (2.0 M, $10.8 \mathrm{~mL}, 21.6$ mmole). After 15 mins the solution was cooled to $-78^{\circ} \mathrm{C}$ and a solution of ethyl-2-methoxy-6-methyl benzoate ${ }^{1}$ (3.00 g, 15.45 mmole) and THF (30 mL) was added via cannula. The resulting red solution was stirred for $2 \mathrm{hrs}$ at $-78^{\circ} \mathrm{C}$ before $\mathrm{Bu}_{3} \mathrm{SnCl}(5.44 \mathrm{~mL}, 20.1 \mathrm{mmole})$ was added via a syringe. The resulting yellow solution was warmed to room temperature and quenched with aq $\mathrm{NH}_{4} \mathrm{Cl}$. The product was extracted with $\mathrm{Et}_{2} \mathrm{O}$ (x3), washed with brine, dried over $\mathrm{MgSO}_{4}$, filtered and concentrated. Purification by flash chromatography (25:1 Hexanes:EtOAc) yielded 4.57 g, $(61 \%)$ of stannane as a clear colourless liquid. ${ }^{1} \mathrm{H}$ NMR $(300 \mathrm{MHz}) \delta 7.10(1 \mathrm{H}, \mathrm{t}, J=$ $8.0 \mathrm{~Hz}), 6.58(1 \mathrm{H}, \mathrm{d}, J=7.8 \mathrm{~Hz}), 6.51(1 \mathrm{H}, \mathrm{d}, J=8.3 \mathrm{~Hz}), 4.34(2 \mathrm{H}, \mathrm{q}, J=7.1 \mathrm{~Hz}), 3.76$ $(3 \mathrm{H}, \mathrm{s}), 2.22$ (2H, s), 1.43-1.16 (15H, m), 0.92-0.72 (15H, m); ${ }^{13} \mathrm{C}$ NMR $(75 \mathrm{MHz}) \delta$ 168.5, 156.6, 142.9, 129.8, 121.2, 120.5, 120.4, 105.5, 60.7, 55.6, 28.8 (t, $J=10 \mathrm{~Hz}$ ), 27.2 (t, $J=27.6$ and 34.6 Hz), 17.4, 16.2, 13.6, 9.8 (t, $J=153.6$ and 160.3 Hz); LR-EIMS m/z: $427\left(\mathrm{M}^{+}-\mathrm{C}_{4} \mathrm{H}_{9}, 100\right), 425\left(\mathrm{M}^{+}-\mathrm{C}_{4} \mathrm{H}_{9}, 75\right)$; LR-CIMS $\left(\mathrm{NH}_{3}\right): 485\left(\mathrm{MH}^{+}, 100\right)$; HREIMS calculated for $\mathrm{C}_{19} \mathrm{H}_{31}{ }^{116} \mathrm{SnO}_{3}\left(\mathrm{M}^{+}-\mathrm{C}_{4} \mathrm{H}_{9}\right)$ : 423.1285 found 423.1284 
General Ring Annulation Protocol: To a cooled $\left(-78^{\circ} \mathrm{C}\right)$ solution of stannane $(1.4 \mathrm{eq})$ in THF was added $n$-BuLi (1.4 eq) resulting in a deep red colour. The mixture was stirred for 30 mins before a solution of enone (1.0 eq) and THF was added via cannula. The solution was stirred at $-78^{\circ} \mathrm{C}$ for $1 \mathrm{hr}$ before being warmed to room temperature and stirred for a further 3 hrs (the red colour disappears resulting in a yellow solution). The reaction was quenched with aq. $\mathrm{NH}_{4} \mathrm{Cl}$, extracted with $\mathrm{Et}_{2} \mathrm{O}$ (x3), washed with brine, dried over $\mathrm{MgSO}_{4}$, filtered and concentrated. Purification by flash chromatography (8:1 Hexanes: EtOAc) yielded pure products.<smiles>COc1cccc2c1C(O)=C1C(=O)CCCC1C2</smiles>

Compound 2: Stannane (500 mg, 1.03 mmole) in THF (8 mL), n-BuLi (2.0 M, $520 \mu \mathrm{L}$, 1.03 mmole), cyclohex-1-en-one (71 $\mu \mathrm{L}, 0.739 \mathrm{mmole})$ in THF (2 mL) yielded $135.4 \mathrm{mg}$ (75\%) of product as a pale yellow solid. ${ }^{1} \mathrm{H}$ NMR $(300 \mathrm{MHz}) \delta 7.30(1 \mathrm{H}, \mathrm{t}, J=8.0 \mathrm{~Hz})$, $6.82(1 \mathrm{H}, \mathrm{d}, J=8.5 \mathrm{~Hz}), 6.73(1 \mathrm{H}, \mathrm{d}, J=7.6 \mathrm{~Hz}), 3.87(3 \mathrm{H}, \mathrm{s}), 2.69$ (1H, dd, $J=13.2,3.0$ $\mathrm{Hz}), 2.62-2.53(1 \mathrm{H}, \mathrm{m}), 2.59$ (1H, d, $J=13.2 \mathrm{~Hz}), 2.42-2.36$ (2H, m), 1.99-1.83 (2H, m), 1.62-1.52 (1H, m), 1.32-1.19 (1H, m); ${ }^{13} \mathrm{C}$ NMR (75 MHz) $\delta 186.9,182.7,159.9,144.6$, 133.3, 120.6, 120.0, 110.4, 109.3, 55.9, 37.5, 32.9, 31.2, 29.9, 20.7; LR-EIMS m/z: 244 ( $\left.\mathrm{M}^{+}, 100\right), 216$ (37), 188 (42); HR-EIMS: calculated for $\mathrm{C}_{15} \mathrm{H}_{16} \mathrm{O}_{3}: 244.1099$ found 244.1099.

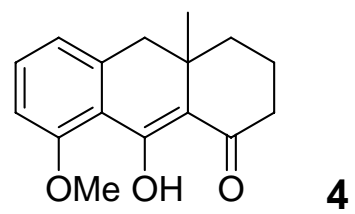

Compound 4: Stannane (500 mg, 1.03 mmole) in THF (8 mL), $n$-BuLi (2.0 M, $520 \mu \mathrm{L}$, 1.03 mmole), 3-methyl-cyclohex-1-en-one (83 $\mu \mathrm{L}, 0.735 \mathrm{mmole}$ ) in THF (2 mL) yielded $140 \mathrm{mg}(74 \%)$ of product as a pale yellow solid. ${ }^{1} \mathrm{H}$ NMR $(300 \mathrm{MHz}) \delta 7.32(1 \mathrm{H}, \mathrm{t}, J=$ $8.0 \mathrm{~Hz}), 6.84(1 \mathrm{H}, \mathrm{d}, J=8.5 \mathrm{~Hz}), 6.73(1 \mathrm{H}, \mathrm{d}, J=7.4 \mathrm{~Hz}), 3.89(3 \mathrm{H}, \mathrm{s}), 2.80(1 \mathrm{H}, \mathrm{d}, J=$ $15.0 \mathrm{~Hz}), 2.55(1 \mathrm{H}, \mathrm{d}, J=15.0 \mathrm{~Hz}), 2.43-2.38(2 \mathrm{H}, \mathrm{m}), 1.86-1.66(3 \mathrm{H}, \mathrm{m}), 1.51(1 \mathrm{H}, \mathrm{dt}, J$ $=13.0,3.8 \mathrm{~Hz}), 0.95(3 \mathrm{H}, \mathrm{s}) ;{ }^{13} \mathrm{C}$ NMR $(75 \mathrm{MHz}) \delta 186.3,182.8,159.9,143.4,133.5$, 120.8, 119.9, 112.8, 110.4, 55.8, 45.1, 37.2, 32.8, 31.0, 24.7, 17.2; LR-EIMS m/z: 258 $\left(\mathrm{M}^{+}, 20\right), 243\left(\mathrm{M}^{+}-\mathrm{CH}_{3}, 100\right)$; HR-EIMS: calculated for $\mathrm{C}_{16} \mathrm{H}_{18} \mathrm{O}_{3}$ : 258.1256 found 258.1252.<smiles>COc1cccc2c1C(O)=C1C(=O)CC(C)(C)CC1(C)C2</smiles> 
Compound 5: Stannane (500 mg, 1.03 mmole) in THF (8 mL), $n$-BuLi (2.0 M, $520 \mu \mathrm{L}$, 1.03 mmole), isophorone ( $110 \mu \mathrm{L}, 0.735 \mathrm{mmole})$ in THF ( $2 \mathrm{~mL}$ ) yielded $175.3 \mathrm{mg}(83 \%)$ of product as a white solid. ${ }^{1} \mathrm{H}$ NMR $(300 \mathrm{MHz}) \delta 7.27(1 \mathrm{H}, \mathrm{t}, J=8.0 \mathrm{~Hz}), 6.79(1 \mathrm{H}, \mathrm{d}, J$ $=8.4 \mathrm{~Hz}), 6.68(1 \mathrm{H}, \mathrm{d}, J=7.4 \mathrm{~Hz}), 3.85(3 \mathrm{H}, \mathrm{s}), 2.86(1 \mathrm{H}, \mathrm{d}, J=15.2 \mathrm{~Hz}), 2.47(1 \mathrm{H}, \mathrm{d}, J$ $=15.2 \mathrm{~Hz}), 2.17(2 \mathrm{H}, \mathrm{d}, J=2.17 \mathrm{~Hz}), 1.50(2 \mathrm{H}, \mathrm{ABq}, J=4.0 \mathrm{~Hz}), 1.02(3 \mathrm{H}, \mathrm{s}), 0.98(3 \mathrm{H}$, s), 0.97 (3H, s); ${ }^{13} \mathrm{C}$ NMR $(75 \mathrm{MHz}) \delta 185.1,183.4,159.9,143.5,133.4,121.0,120.1$, 111.1, 110.3, 55.8, 50.5, 46.4, 45.7, 33.1, 30.8, 30.5, 30.0, 27.2; LR-EIMS m/z: $286\left(\mathrm{M}^{+}\right.$, 15), 271 (100); HR-EIMS: calculated for $\mathrm{C}_{18} \mathrm{H}_{22} \mathrm{O}_{3}$ : 286.1569 found 286.1569.

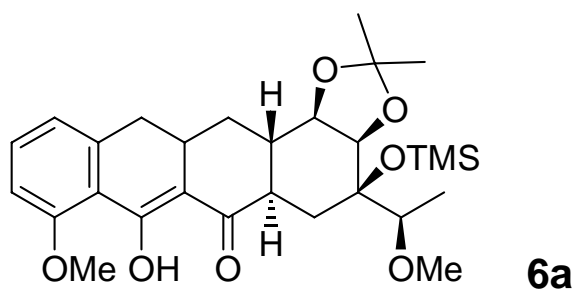

Compound 6a: Stannane (244 mg, 0.505 mmole) in THF ( $5 \mathrm{~mL}$ ), $n$-BuLi (2.0 M, 252 $\mu \mathrm{L}, 0.505$ mmole), enone (133 mg, $0.361 \mathrm{mmole})$ in THF (5 mL) yielded $132.9 \mathrm{mg}$ (71\%) of tetracycle as an amorphous solid. ${ }^{1} \mathrm{H}$ NMR $(300 \mathrm{MHz}) \delta 7.34(1 \mathrm{H}, \mathrm{t}, J=8.0$ $\mathrm{Hz}), 6.86(1 \mathrm{H}, \mathrm{d}, J=8.4 \mathrm{~Hz}), 6.78(1 \mathrm{H}, \mathrm{d}, J=7.4 \mathrm{~Hz}), 3.95-3.91(2 \mathrm{H}, \mathrm{m}), 3.91(3 \mathrm{H}, \mathrm{s})$, $3.28(3 \mathrm{H}, \mathrm{s}), 3.28-3.24(1 \mathrm{H}, \mathrm{m}), 2.78-2.47(5 \mathrm{H}, \mathrm{m}), 2.27-2.22(1 \mathrm{H}, \mathrm{m}), 1.59-1.53(2 \mathrm{H}$, m), 1.47 (3H, s), 1.39 (3H, s), $1.27(3 \mathrm{H}, \mathrm{d}, J=6.2 \mathrm{~Hz}), 1.04(1 \mathrm{H}, \mathrm{q}, J=11.1 \mathrm{~Hz}), 0.12$ (9H, s); ${ }^{13} \mathrm{C}$ NMR $(75 \mathrm{MHz}) \delta 186.1,185.4,159.8,144.5,133.2,120.6,120.0,110.5$, 108.4, 107.5, 107.3, 85.3, 80.5, 80.4, 77.8, 57.2, 56.0, 40.3, 38.9, 37.6, 33.3, 32.3, 30.6, 28.7, 26.3, 12.5, 2.7; LR-EIMS m/z: $516\left(\mathrm{M}^{+}, 75\right), 399\left(\mathrm{M}^{+}-\mathrm{C}_{6} \mathrm{H}_{13} \mathrm{O}_{2}, 100\right), 457\left(\mathrm{M}^{+}-\right.$ $\mathrm{C}_{3} \mathrm{H}_{7} \mathrm{O}$, 90); HR-EIMS calculated for $\mathrm{C}_{28} \mathrm{H}_{40} \mathrm{O}_{7} \mathrm{Si}$ : 516.2543 found 516.2555.

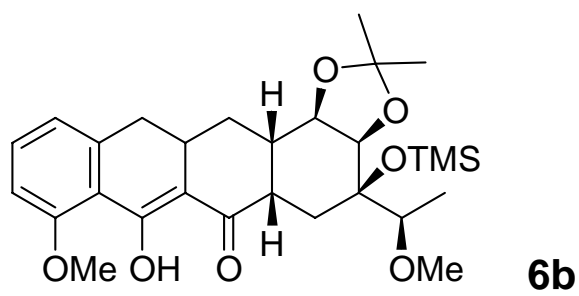

Compound 6b: Stannane (354 mg, 0.732 mmole) in THF (7.5 mL), n-BuLi (2.0 M, 370 $\mu \mathrm{L}, 0.732 \mathrm{mmole}$ ), enone (192.7 mg, $0.523 \mathrm{mmole}$ ) in THF (7.5 mL) yielded $167.7 \mathrm{mg}$ (62\%) of tetracycle as an amorphous solid. ${ }^{1} \mathrm{H}$ NMR $(300 \mathrm{MHz}) \delta 7.31(1 \mathrm{H}, \mathrm{t}, J=8.0$ $\mathrm{Hz}), 6.83(1 \mathrm{H}, \mathrm{d}, J=8.4 \mathrm{~Hz}), 6.73(1 \mathrm{H}, \mathrm{d}, J=7.4 \mathrm{~Hz}), 4.03(1 \mathrm{H}, \mathrm{d}, J=7.1 \mathrm{~Hz}), 3.98-$ $3.90(1 \mathrm{H}, \mathrm{m}), 3.88(3 \mathrm{H}, \mathrm{s}), 3.24-3.17(1 \mathrm{H}, \mathrm{m}), 3.22(3 \mathrm{H}, \mathrm{s}), 2.85-2.66(3 \mathrm{H}, \mathrm{m}), 2.52(1 \mathrm{H}$, d, $J=14.1 \mathrm{~Hz}$ ), 2.45-2.29 (2H, m), 2.16-2.12 (1H, m), 1.49 (3H, s), 1.45-1.35 (2H, m), 1.29 (3H, s), 1.17 (3H, d, $J=6.2 \mathrm{~Hz}), 0.12(9 \mathrm{H}, \mathrm{s}) ;{ }^{13} \mathrm{C}$ NMR $(75 \mathrm{MHz}) \delta 186.3,185.9$, $159.8,144.5,133.3,120.8,120.0,110.5,108.5,108.3,81.0,77.7,75.7,74.5,56.2$, 56.0, 37.5, 36.5, 33.0, 29.6, 29.5, 28.0, 27.4, 25.2, 12.3, 2.7; LR-EIMS m/z: $516\left(\mathrm{M}^{+}, 10\right), 457$ $\left(\mathrm{M}^{+}-\mathrm{C}_{3} \mathrm{H}_{7} \mathrm{O}, 100\right)$, $399\left(\mathrm{M}^{+}-\mathrm{C}_{6} \mathrm{H}_{13} \mathrm{O}_{2}, 35\right)$; HR-EIMS: calculated for $\mathrm{C}_{28} \mathrm{H}_{40} \mathrm{O}_{7} \mathrm{Si}$ : 516.2543 found 516.2531 . 


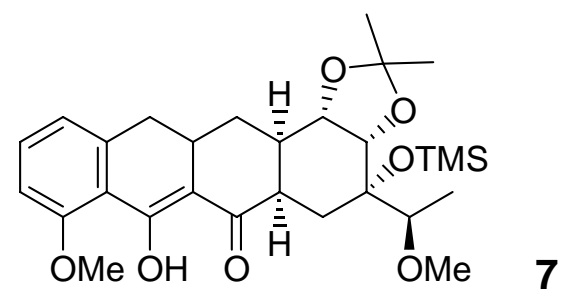

Compound 7: Stannane (228 mg, $0.472 \mathrm{mmole}$ ) in THF ( $5 \mathrm{~mL}$ ), $n$-BuLi (2.0 M, $240 \mu \mathrm{L}$, $0.472 \mathrm{mmole}$ ), enone (124.3 mg, $0.337 \mathrm{mmole}$ ) in THF ( $5 \mathrm{~mL}$ ) yielded $89.6 \mathrm{mg}(51 \%)$ of tetracycle as an amorphous solid. ${ }^{1} \mathrm{H}$ NMR $(300 \mathrm{MHz}) \delta 7.31(1 \mathrm{H}, \mathrm{t}, J=8.0 \mathrm{~Hz}), 6.83$ $(1 \mathrm{H}, \mathrm{d}, J=8.4 \mathrm{~Hz}$ ), $6.73(1 \mathrm{H}, \mathrm{d}, J=7.4 \mathrm{~Hz}), 4.04-3.92(2 \mathrm{H}, \mathrm{m}), 3.88(3 \mathrm{H}, \mathrm{s}), 3.26-3.22$ (1H, m), 3.24 (3H, s), 2.85-2.66 (3H, m), 2.55-2.46 (2H, m), 2.23-2.13 (2H, m), $1.65(1 \mathrm{H}$, dd, $J=14.9,8.9 \mathrm{~Hz}), 1.45-1.40(1 \mathrm{H}, \mathrm{m}), 1.44(3 \mathrm{H}, \mathrm{s}), 1.27(3 \mathrm{H}, \mathrm{s}), 1.15$ (3H, d, $J=6.2$ $\mathrm{Hz}), 0.13(9 \mathrm{H}, \mathrm{s}) ;{ }^{13} \mathrm{C}$ NMR $(75 \mathrm{HMz}) \delta 187.1,184.8,159.8,144.7,135.5,120.8,120.0$, 110.4, 108.6, 108.5, 81.4, 77.9, 74.5, 74.4, 56.3, 56.0, 37.5, 36.3, 33.2, 31.2, 29.7, 28.0, 27.6, 25.6, 13.7, 2.4; LR-EIMS m/z: $516\left(\mathrm{M}^{+}, 5\right), 457$ (100), 399 (25), 367 (20); HREIMS: calculated for $\mathrm{C}_{28} \mathrm{H}_{40} \mathrm{O}_{7} \mathrm{Si} 516.2543$ found 516.2548.

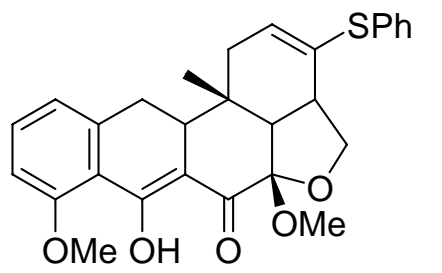

Compound 8: Stannane (62 mg, $0.128 \mathrm{mmole}$ ), in THF ( $5 \mathrm{~mL}), n$-BuLi (2.0 M, $65 \mu \mathrm{L}$, 0.128 mmole), enone (30.1 mg, $0.0916 \mathrm{mmole})$ in THF ( $1 \mathrm{~mL}$ ) yielded $20.6 \mathrm{mg}(47 \%)$ of pentacycle as a film. ${ }^{1} \mathrm{H}$ NMR $(300 \mathrm{MHz}) \delta 7.40-7.22(6 \mathrm{H}, \mathrm{m}), 6.87(1 \mathrm{H}, \mathrm{d}, J=8.5 \mathrm{~Hz})$, $6.77(1 \mathrm{H}, \mathrm{d}, J=8.5 \mathrm{~Hz}), 6.05(1 \mathrm{H}, \mathrm{d}, J=6.7 \mathrm{~Hz}), 4.04(1 \mathrm{H}, \mathrm{t}, J=8.5 \mathrm{~Hz}), 3.92(3 \mathrm{H}, \mathrm{s})$, 3.42 (3H, s), 3.31-3.28 (2H, m), 2.94-2.88 (2H, m), 2.64-2.53 (2H, m), $2.42(1 \mathrm{H}, \mathrm{dd}, J=$ 17.5, $6.9 \mathrm{~Hz}), 2.12-2.02(1 \mathrm{H}, \mathrm{m}), 1.09$ (3H, s); LR-EIMS m/z: $476\left(\mathrm{M}^{+}, 42\right), 444(100)$, 416 (65), 367 (33), 307 (70), 201 (32); HR-EIMS calculated for $\mathrm{C}_{28} \mathrm{H}_{28} \mathrm{O}_{5} \mathrm{~S} 476.1657$ found 476.1656 .

${ }^{1}$ Hauser, F.M.; Pogany, S.A. Synthesis, 1980, 814 and Hamada, Y.; Hara, O.; Kawai, A.; Kohno, Y.; Shioiri, T. Tetrahedron, 1991, 47, 8635 
Stannane 1, $300 \mathrm{MHz}^{1} \mathrm{H}$ NMR $\left(\mathrm{CDCl}_{3}\right)$

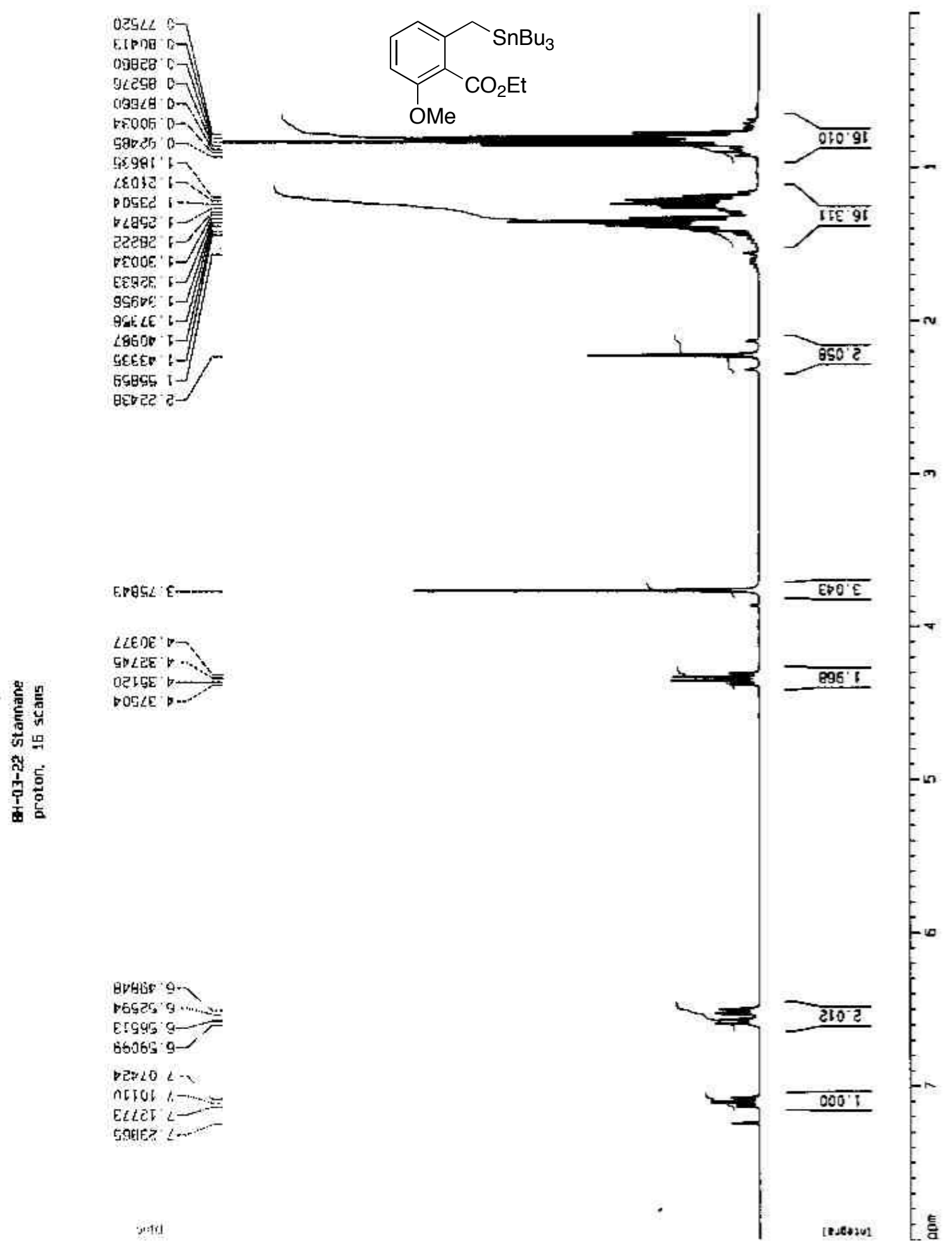


Stannane 1, $75 \mathrm{MHz}^{13} \mathrm{C} \mathrm{NMR}\left(\mathrm{CDCl}_{3}\right)$
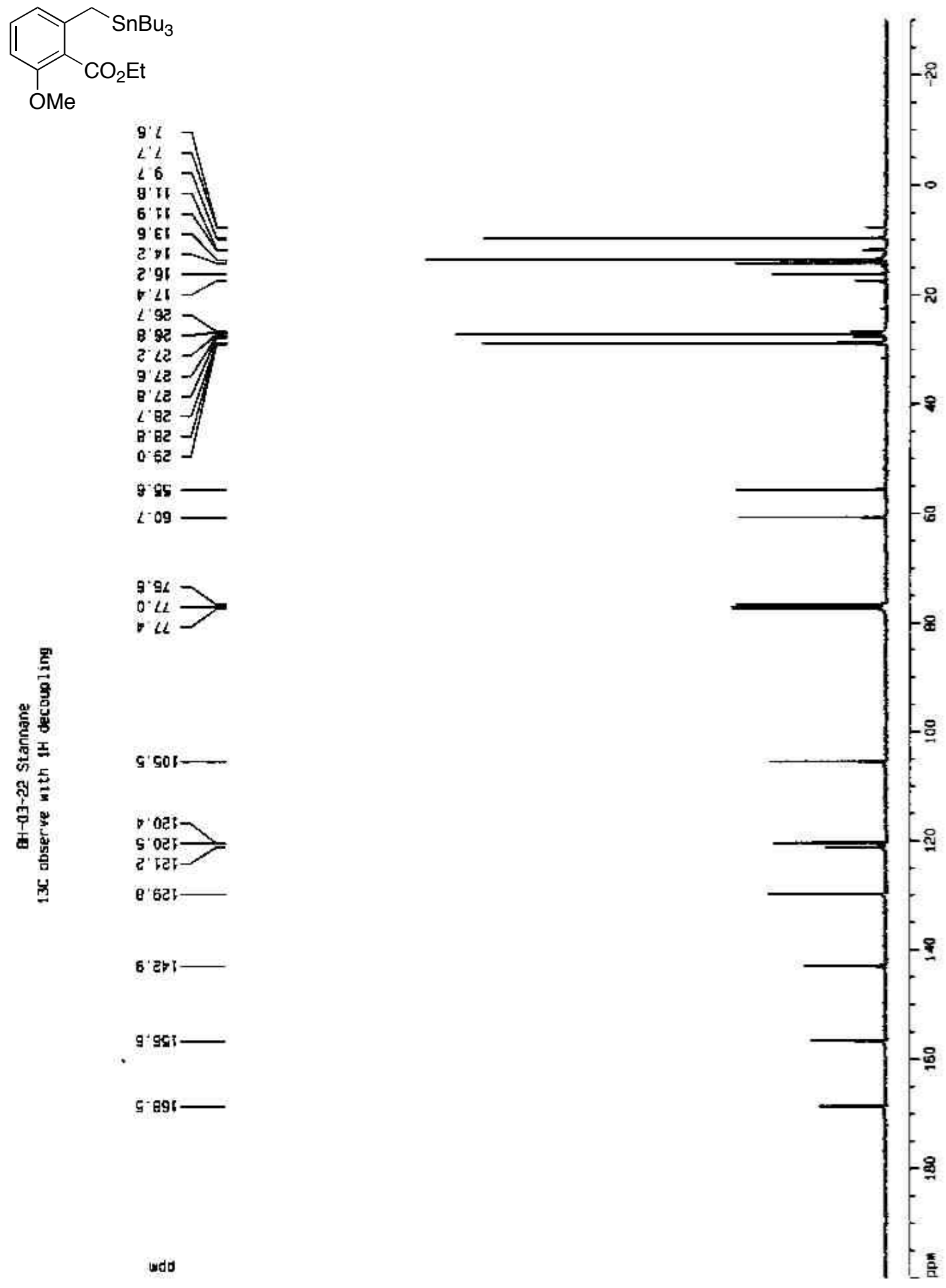
Compound 2, $300 \mathrm{MHz}{ }^{1} \mathrm{H}$ NMR $\left(\mathrm{CDCl}_{3}\right)$

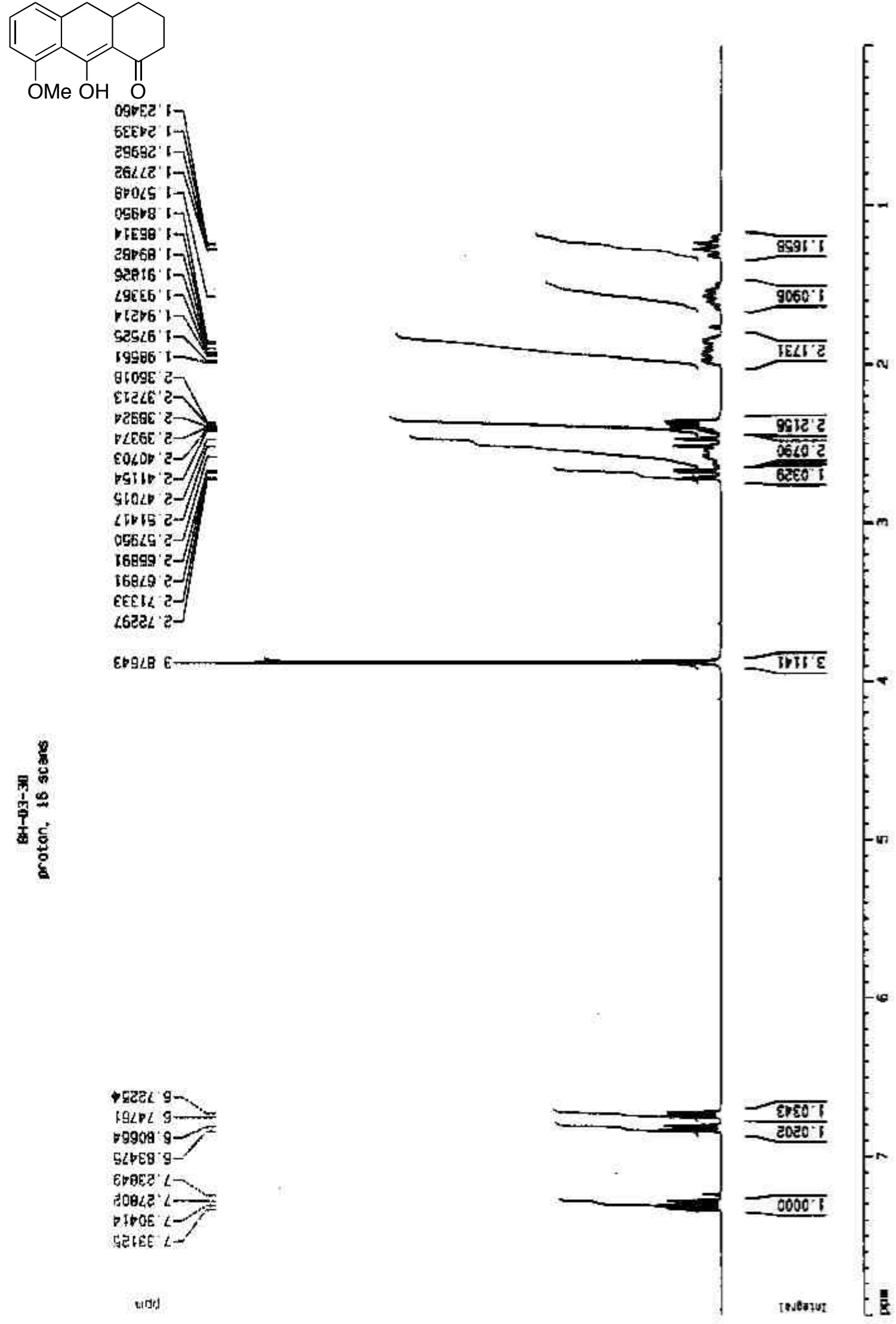


Compound 2, $75 \mathrm{MHz}{ }^{13} \mathrm{C}$ NMR $\left(\mathrm{CDCl}_{3}\right)$<smiles>COc1cccc2c1C(O)=C1C(=O)CCCC1C2</smiles>

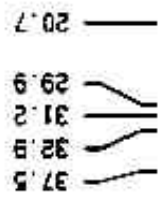

6 '

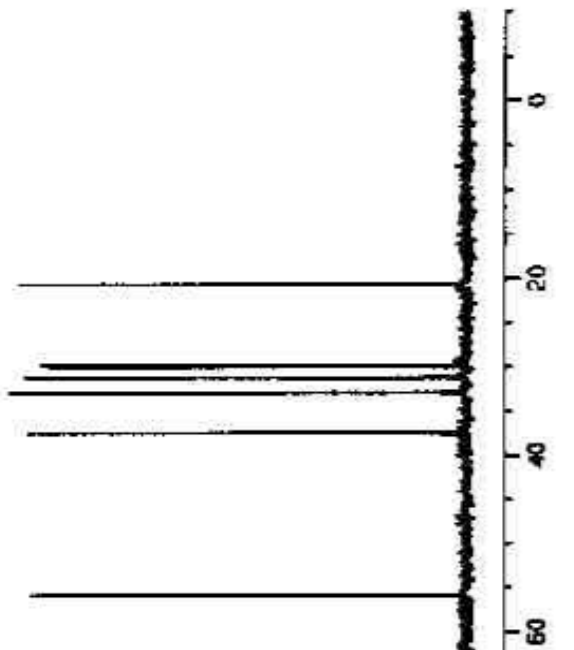

$9.92 \longrightarrow$
$0 \angle 2 \longrightarrow$
$\square L C$

E. $60 \mathrm{H}-2=$

$0.021-2=$
$9021-2=$

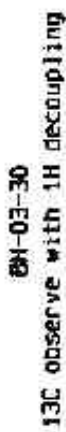

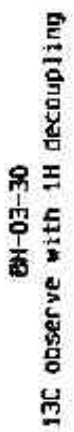

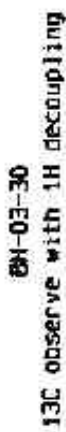

-

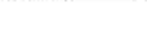

$g \cdot 601$

$6.591-$
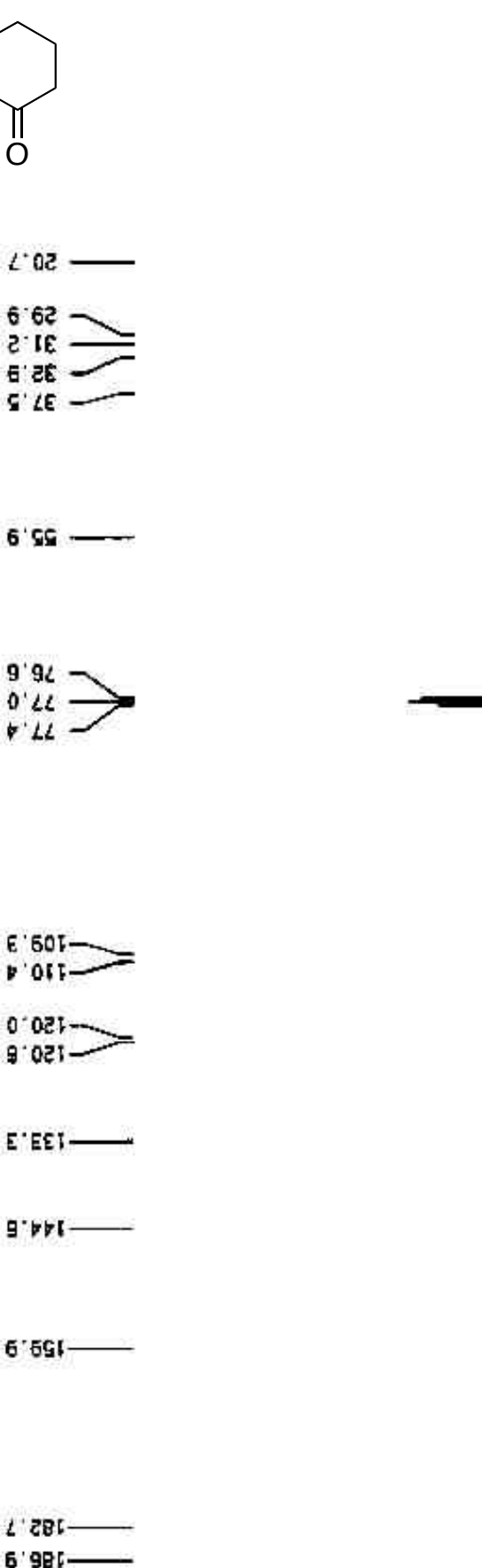

6' $981-$

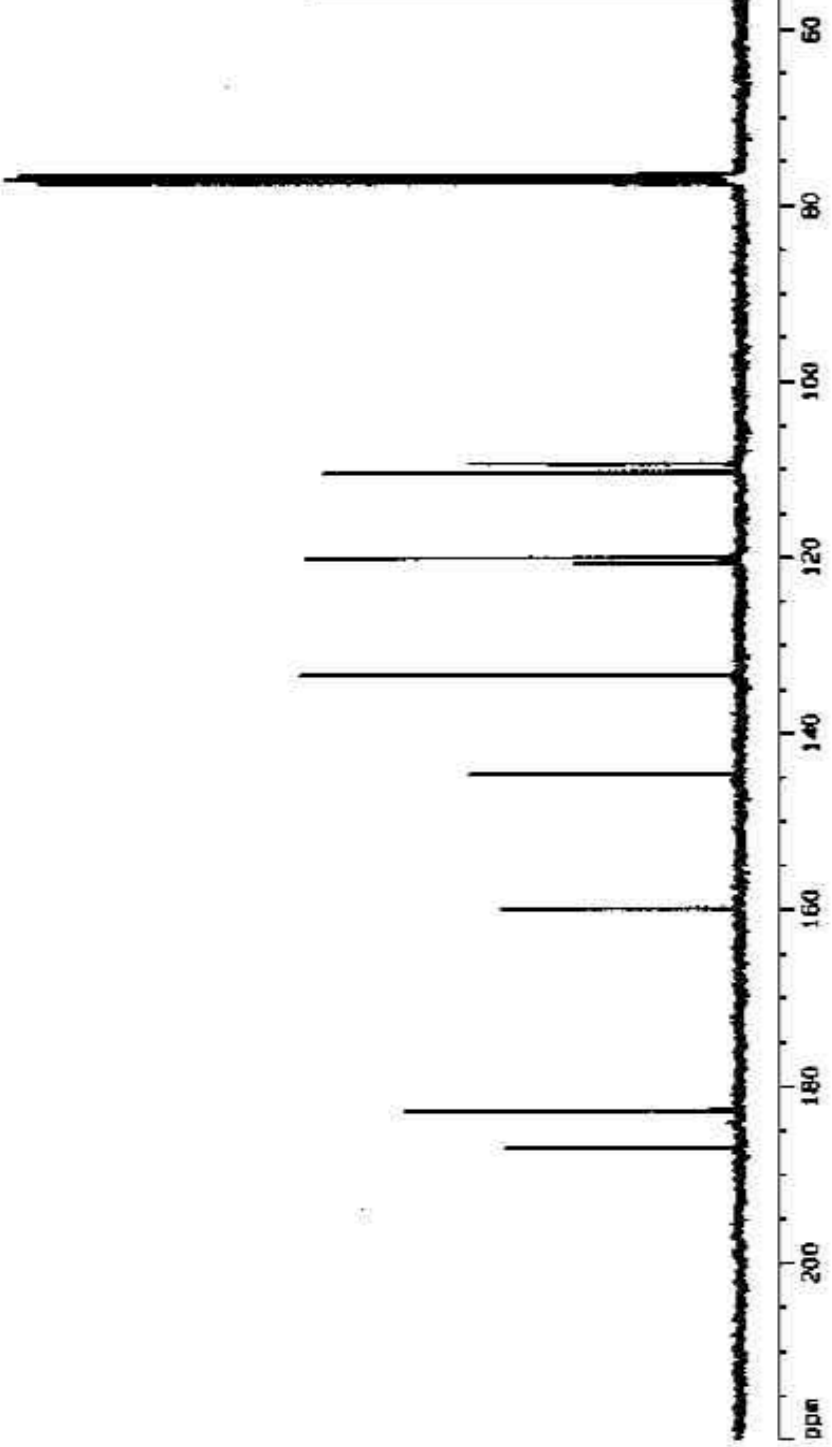




\section{Compound 4, $300 \mathrm{MHz}{ }^{1} \mathrm{H}$ NMR $\left(\mathrm{CDCl}_{3}\right)$}<smiles>COc1cccc2c1C(O)=C1C(=O)CCC[C@]1(C)C2</smiles>

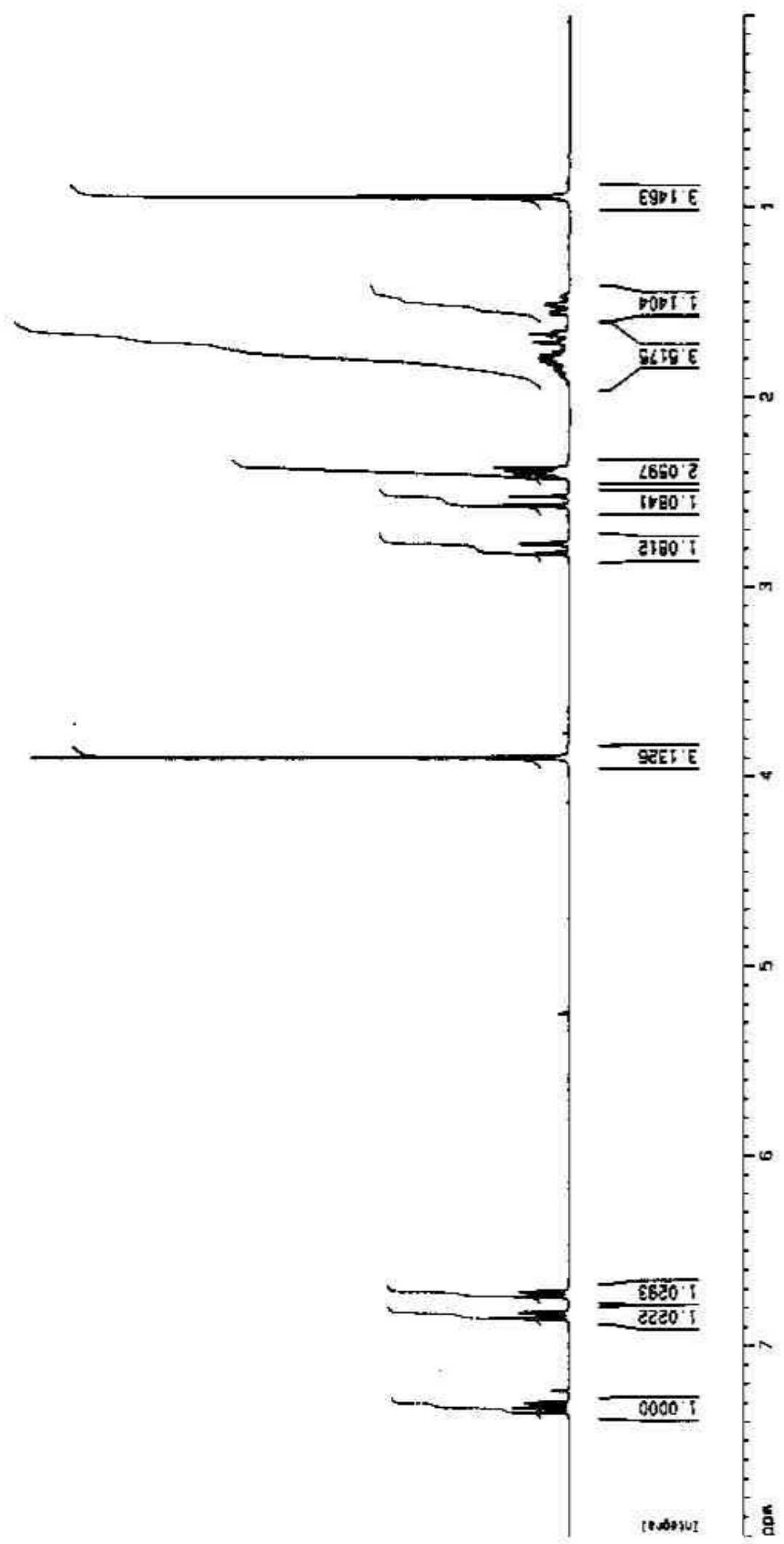


Compound 4, $75 \mathrm{MHz}{ }^{13} \mathrm{C}$ NMR $\left(\mathrm{CDCl}_{3}\right)$<smiles>COc1cccc2c1C(O)=C1C(=O)CCC[C@]1(C)C2</smiles>

$z^{\prime} L$

Liv

O.IE $=$

2. $2 \varepsilon-$

I

8. 5

9.96
0.42
0.4

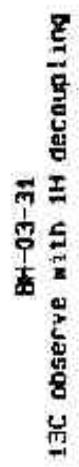

- $051-$

$6.6 \mathrm{H}=$

8 $021-=$

S' $E \varepsilon$

DPL

6.691-
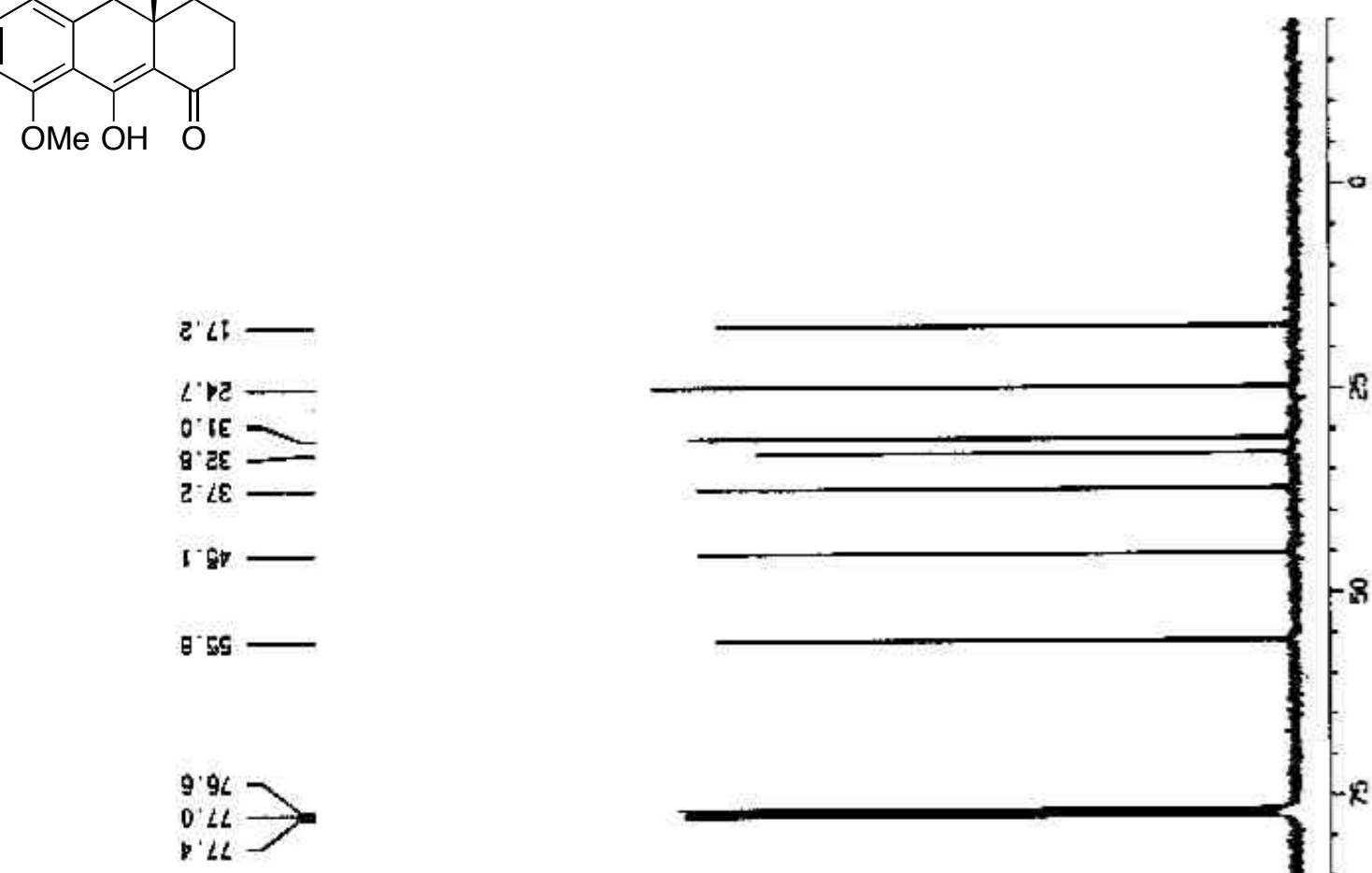

8. $281-$

[.991-

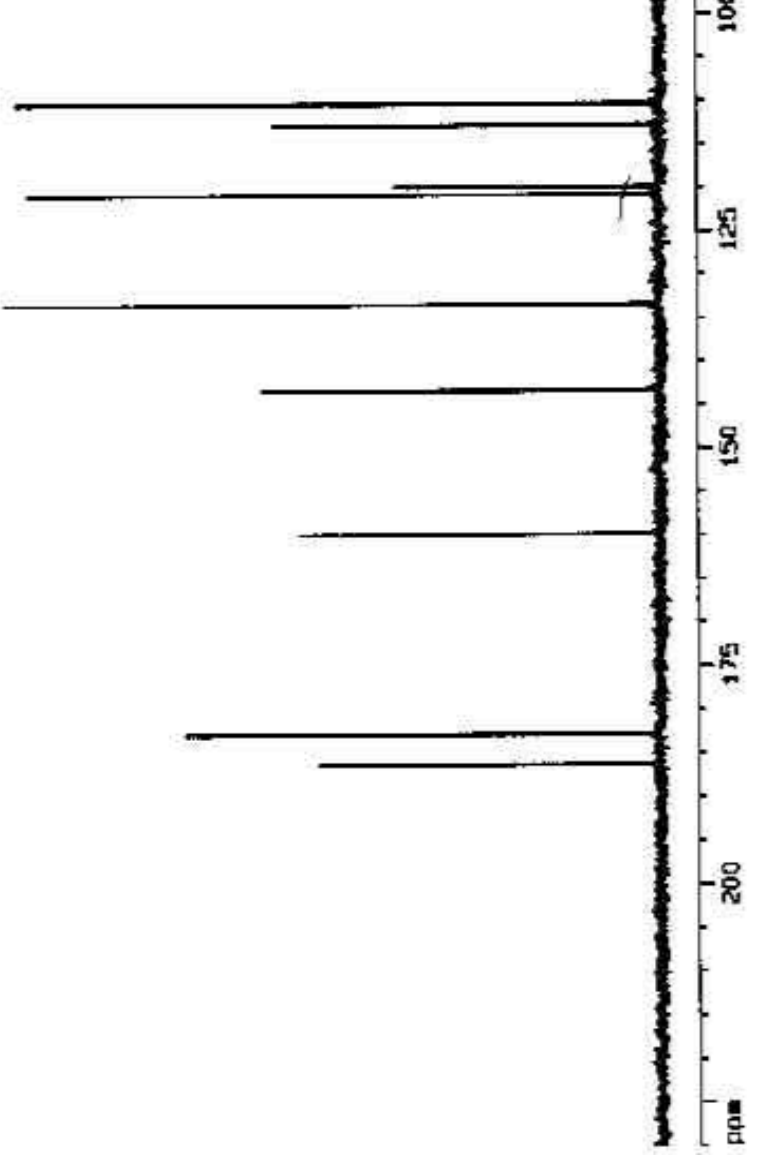




\section{Compound 5, $300 \mathrm{MHz}{ }^{1} \mathrm{H}$ NMR $\left(\mathrm{CDCl}_{3}\right)$}<smiles>COc1cccc2c1C(O)=C1C(=O)CC(C)(C)C[C@]1(C)C2</smiles>

OOC $\angle 6: D-$

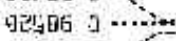

960टी 1-

DS0GF t -2

$90 / 68 \div$

U日E:G $1,+\infty$

SPOOS 1 -

gugat' itv 21 ट $\rightarrow+1=$

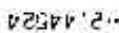

$1696 \mathrm{r}: 3$

566.06 ב 2........

5PRE
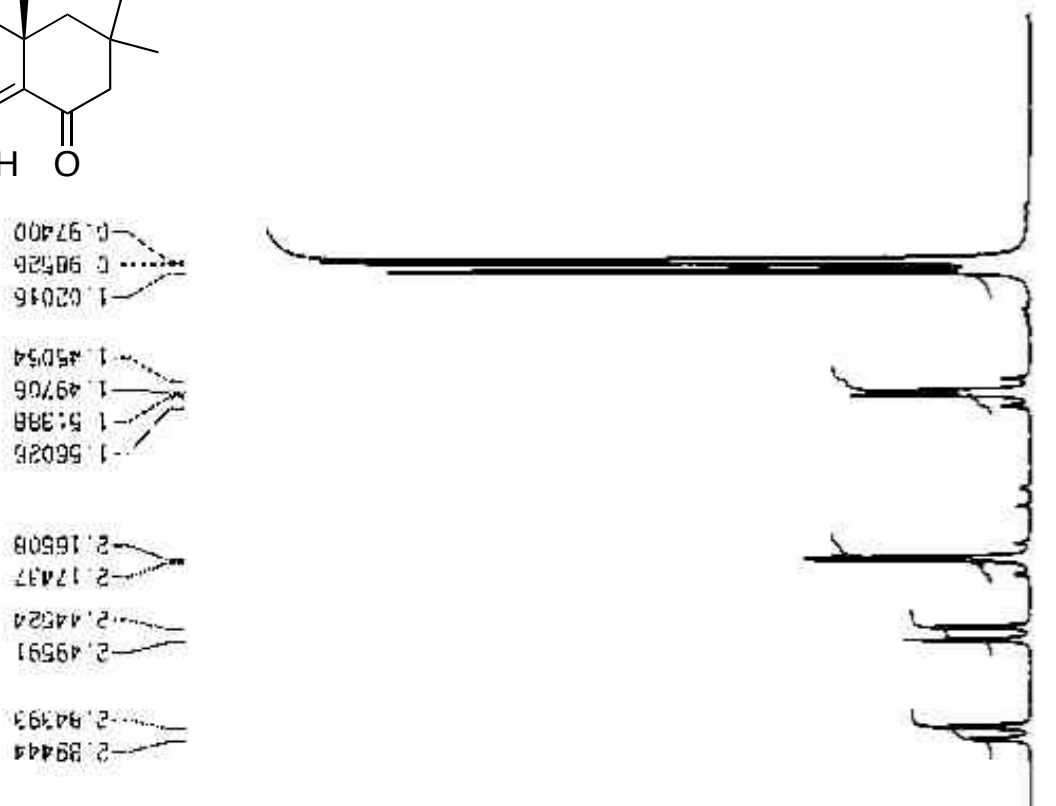

progo: -

点

189- $9-$

v $305 \mathrm{y}$ y-

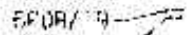

$0 \mathrm{sene}=\mathrm{y}-$

सSEE $t-2$

(1) 可,

$\forall t \in t c^{\prime}+\cdots$

thite: $\angle$.

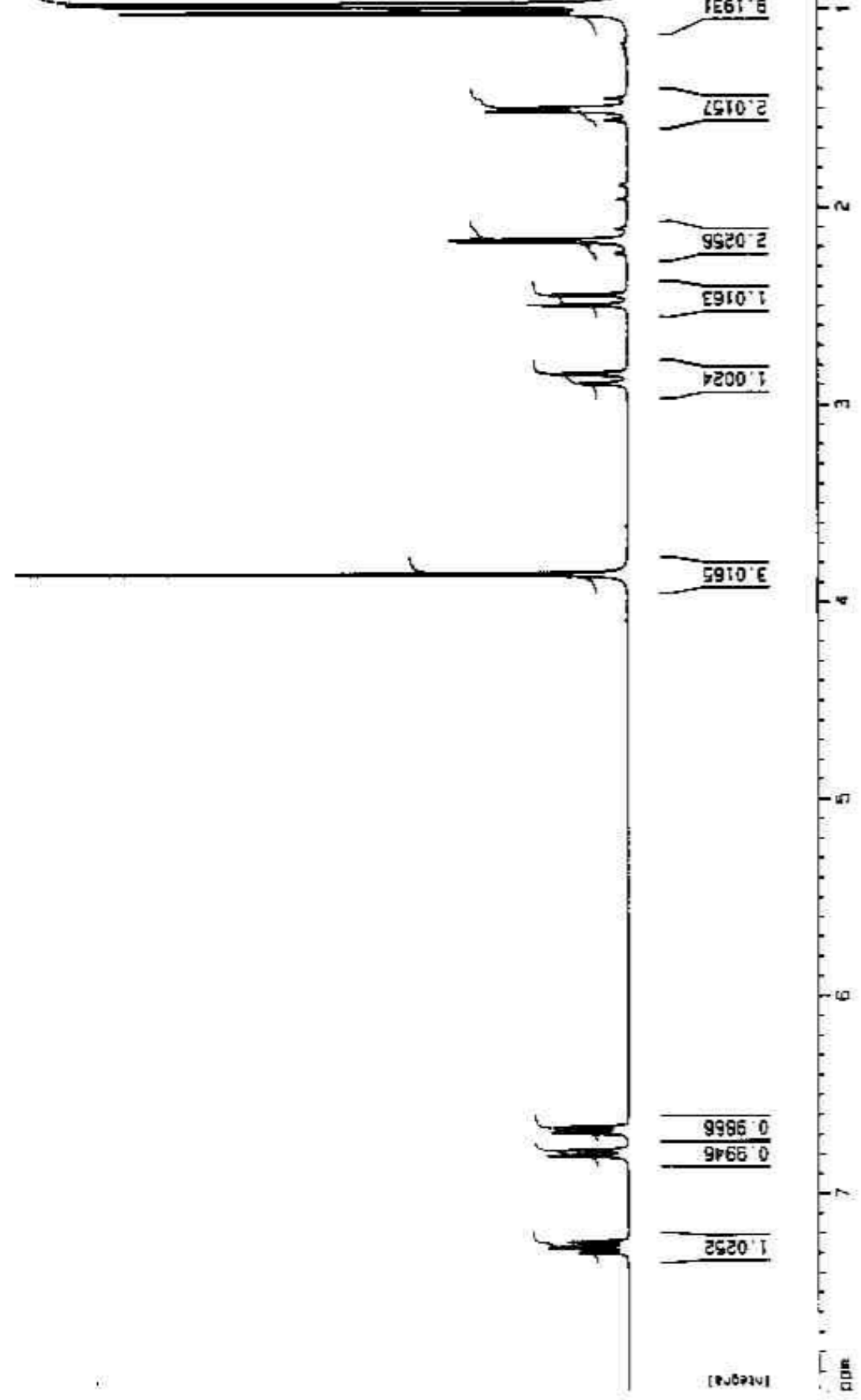


Compound 5, $75 \mathrm{MHz}^{13} \mathrm{C} \mathrm{NMR}\left(\mathrm{CDCl}_{3}\right)$<smiles>COc1cccc2c1C(O)=C1C(=O)CC(C)(C)C[C@]1(C)C2</smiles>
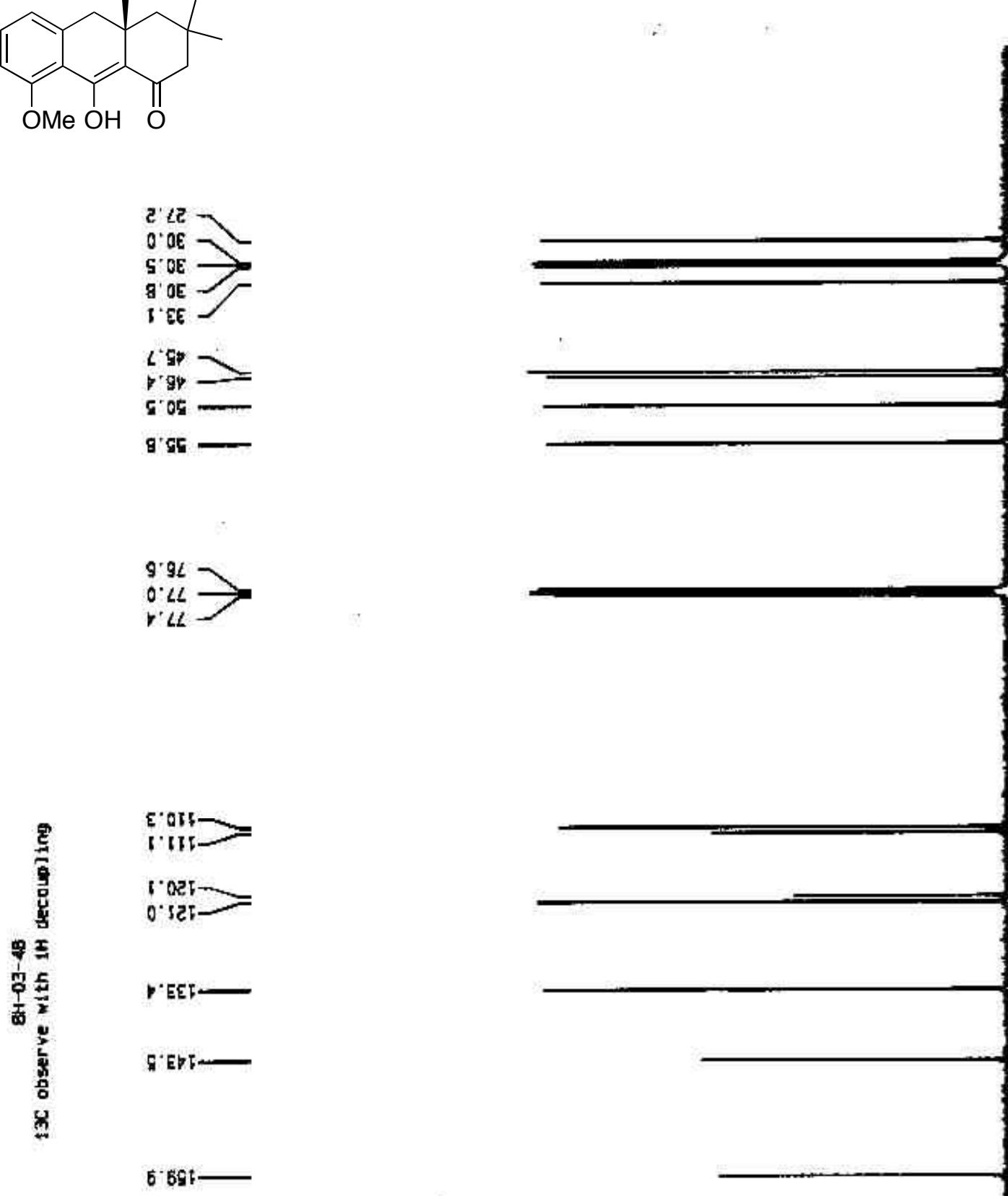

P

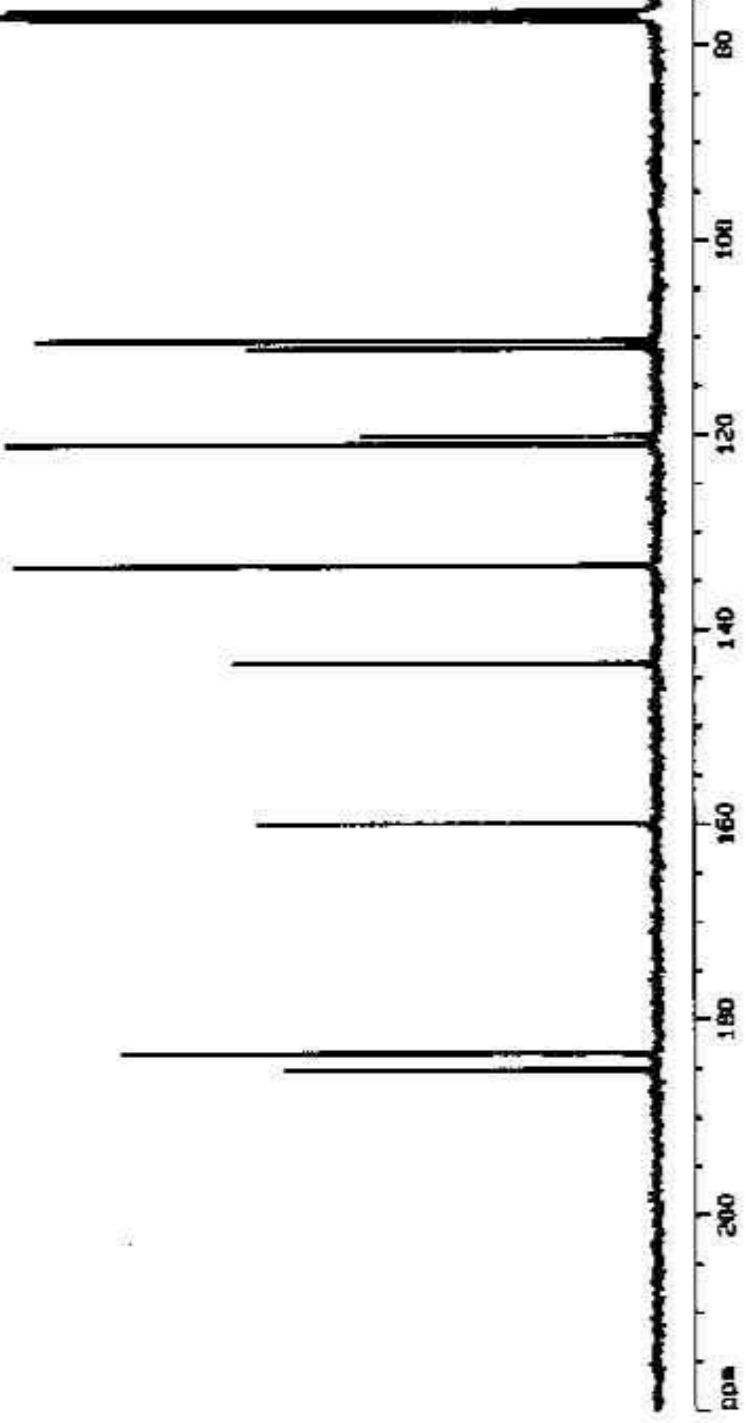


Compound 6a, $300 \mathrm{MHz}^{1} \mathrm{H}$ NMR $\left(\mathrm{CDCl}_{3}\right)$

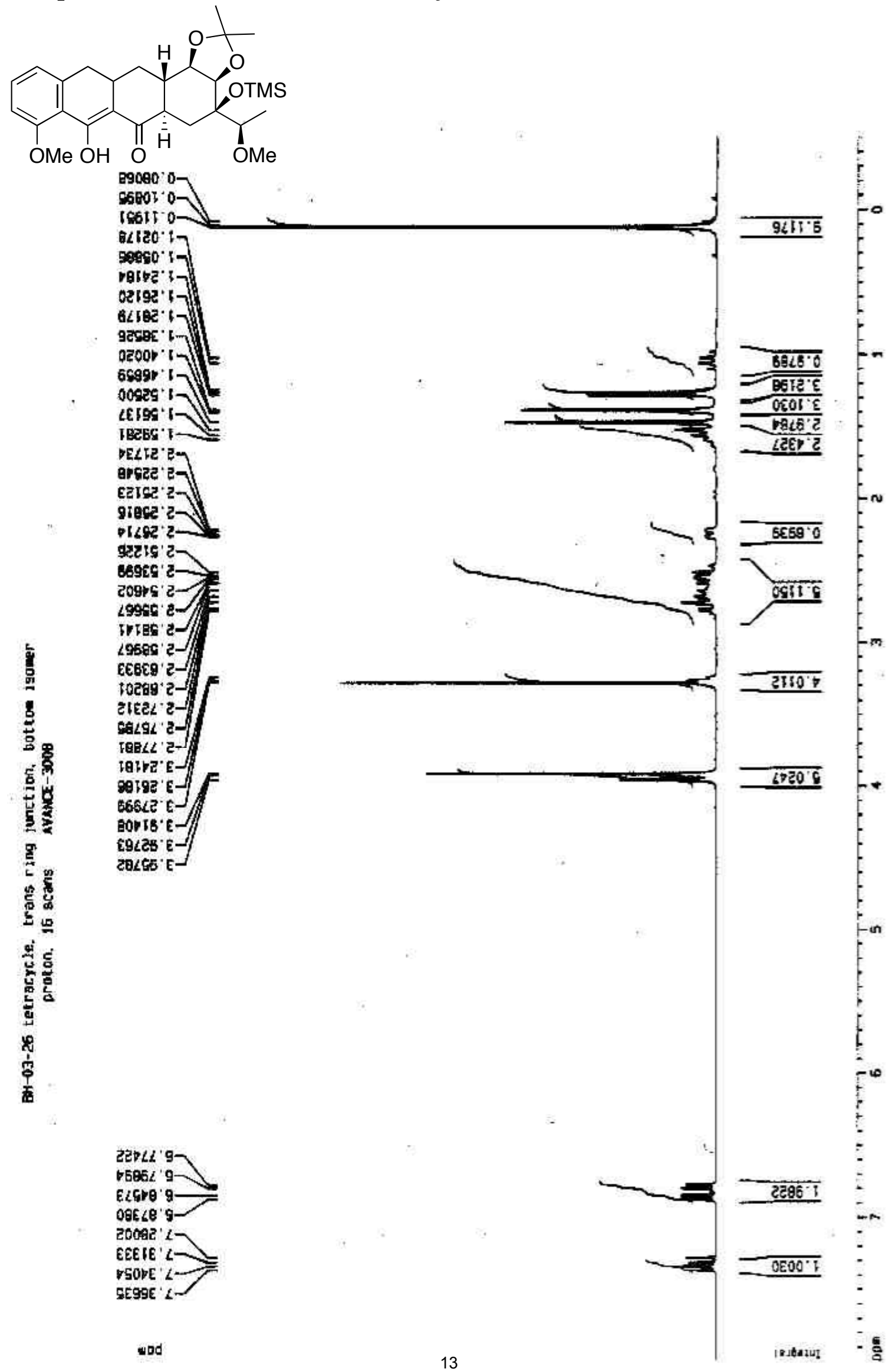




\section{Compound 6a, $75 \mathrm{MHz}^{13} \mathrm{C} \mathrm{NMR}\left(\mathrm{CDCl}_{3}\right)$}<smiles>COc1cccc2c1C(O)=C1C(=O)C3CC(OC(C)=O)(C(C)C)C4OC(C)(C)OC4[C@@H]3CC1CC2</smiles>

IEP 나 -

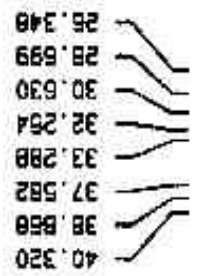

roo $99=$

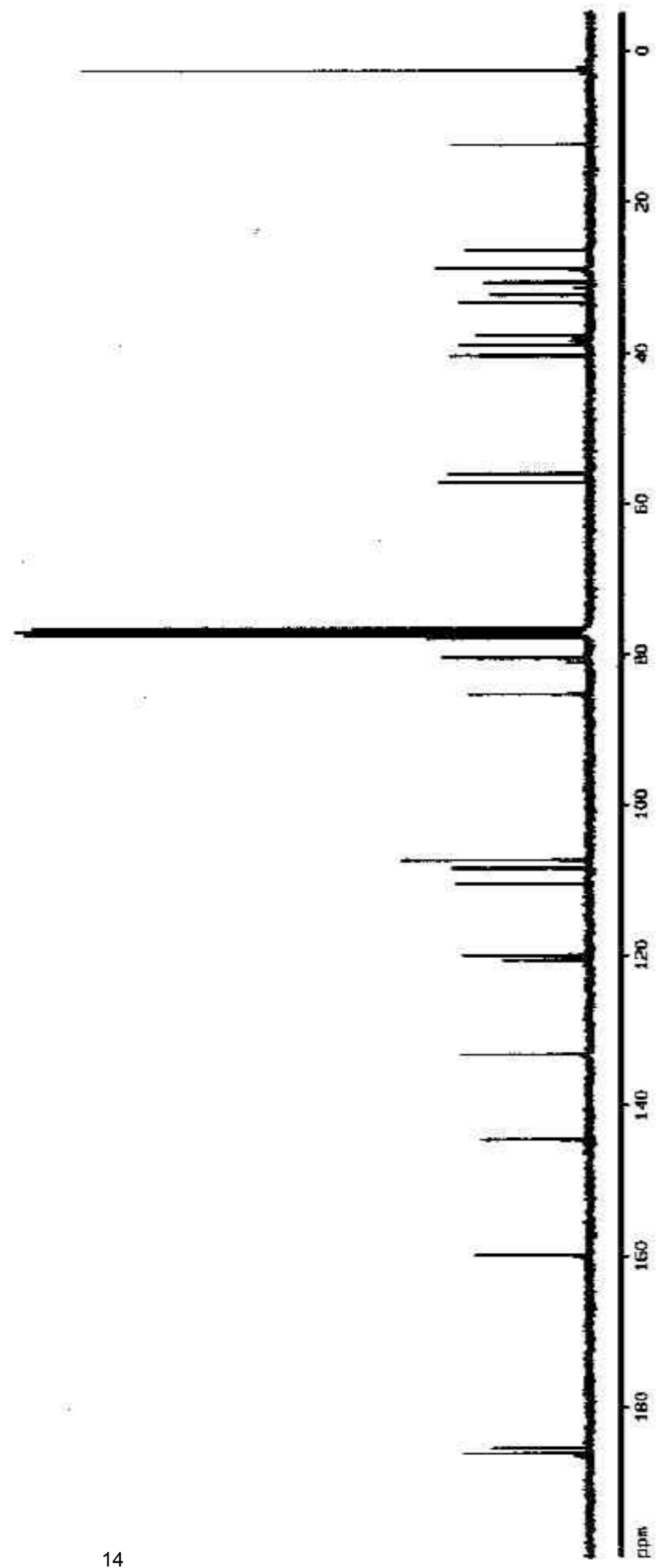

Gic 92

G65' 92

EE⿱ 11

$9: \theta^{\prime} / L \perp$

GSE $08-7$
GAO $08-7$
BLC GE

$\stackrel{5}{*}$

奠

穿

IEE' $201-$

EIS $20 \mathrm{OH}$

ald Bor

$\angle C \mathrm{O} 0 \mathrm{~T} T-$

E

$000,02:-7$

$629.025>$

$661.825-$

要

$\sum_{\square}^{2} \frac{\pi}{5}$

핀

只

客

$\angle E G P D T-$

teg.6st- 
Compound 6b, $300 \mathrm{MHz}^{1} \mathrm{H} \mathrm{NMR}\left(\mathrm{CDCl}_{3}\right)$

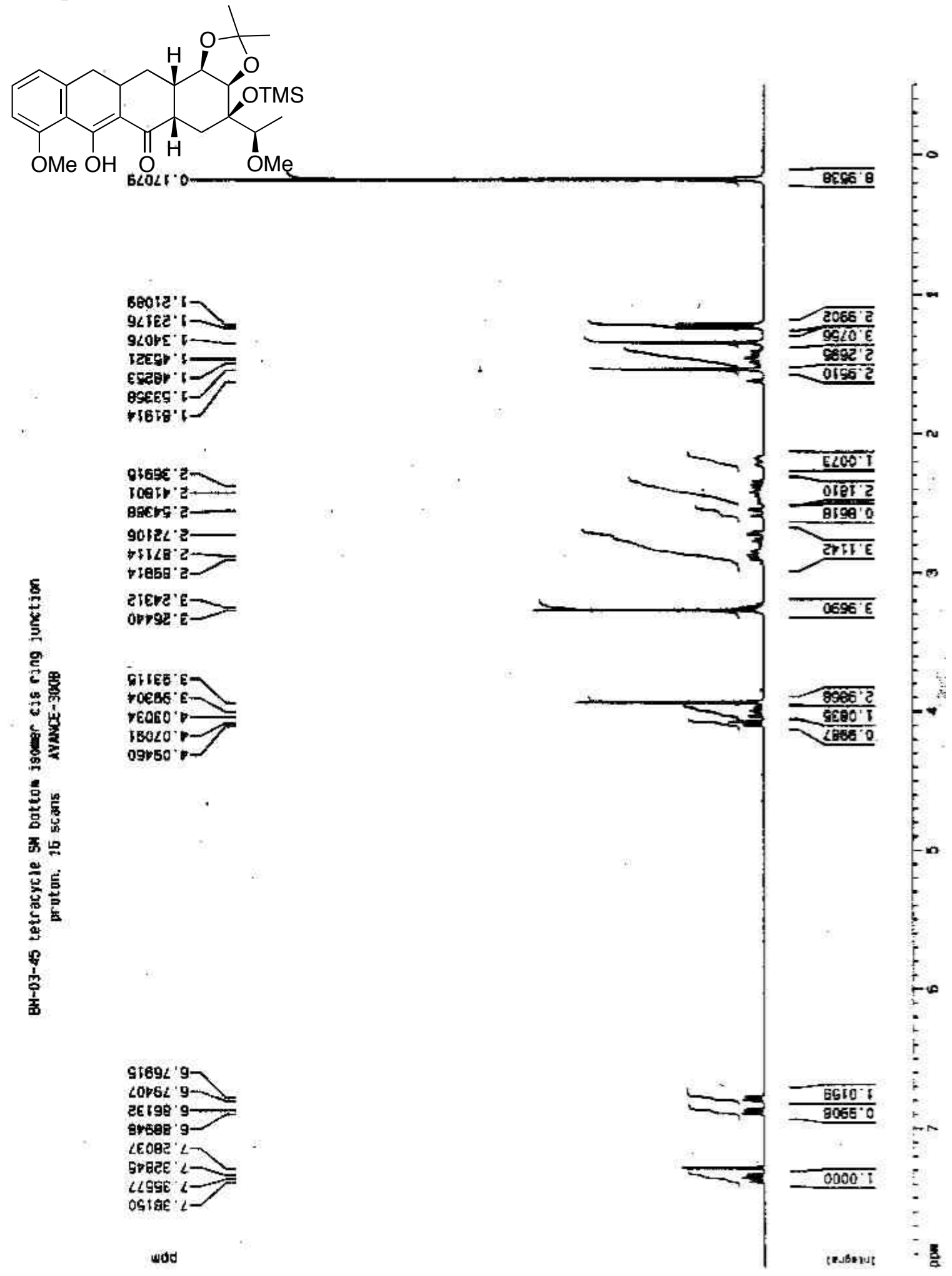


Compound 6b, $75 \mathrm{MHz}^{13} \mathrm{C} \mathrm{NMR}\left(\mathrm{CDCl}_{3}\right)$<smiles>COc1cccc2c1C(O)=C1C(=O)C3CC(C(C)C)(C(C)OC)C4OC(C)(C)OC4C3CC1C2O</smiles>

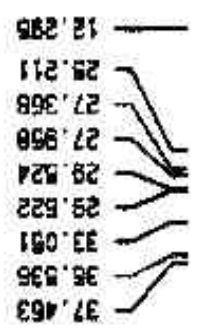

$\sec \cdot 02$
$902 \cdot 95$

Est'

s/9.2 207 000.4

DEv 64

II 20140

8

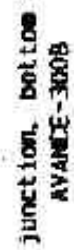

点

c

(1)

i.

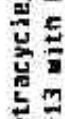

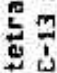

冬

182: $907-$

$160 \cdot 601 \longrightarrow$

$196517-$

QOB OEl

-OE EEI-

Brs $\mathrm{WH}$

0LL 65I-

$P S 8501-2$

เed 981 -

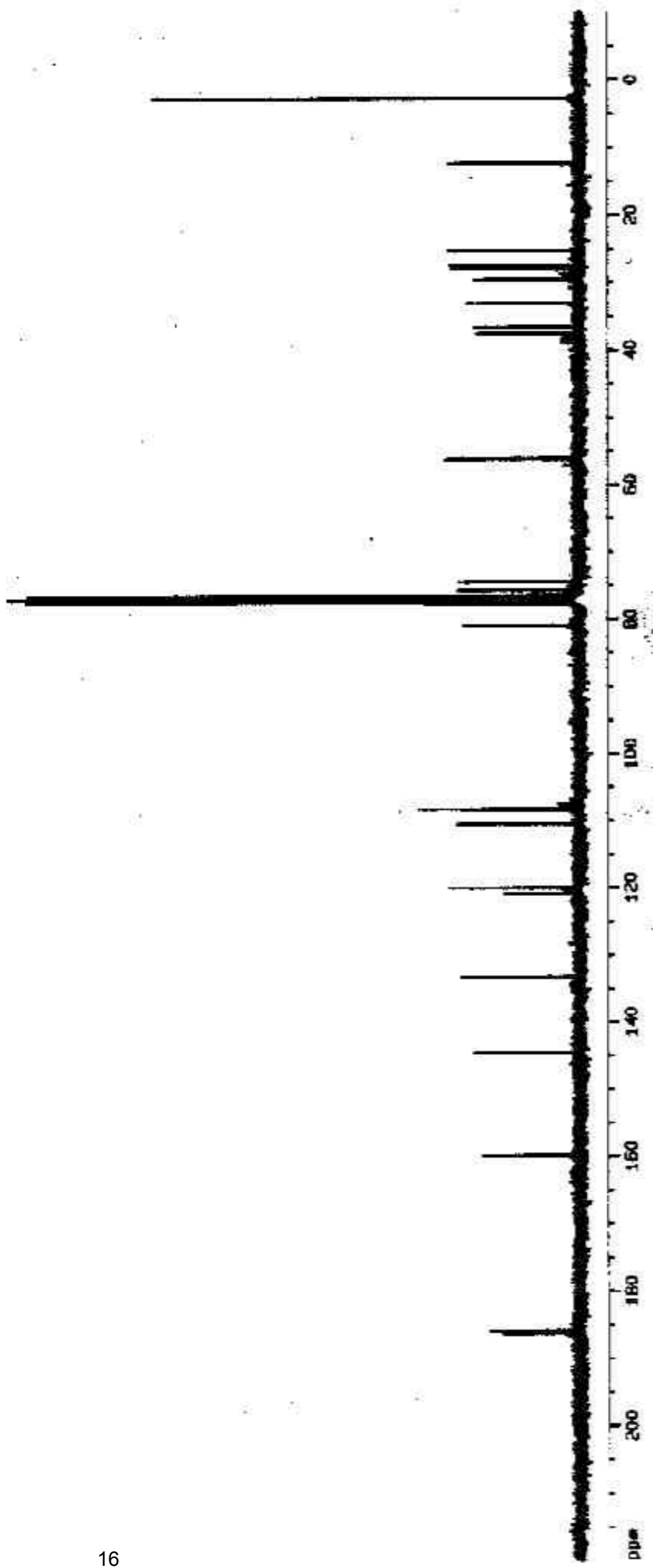




\section{Compound 7, $300 \mathrm{MHz}{ }^{1} \mathrm{H}$ NMR $\left(\mathrm{CDCl}_{3}\right)$}

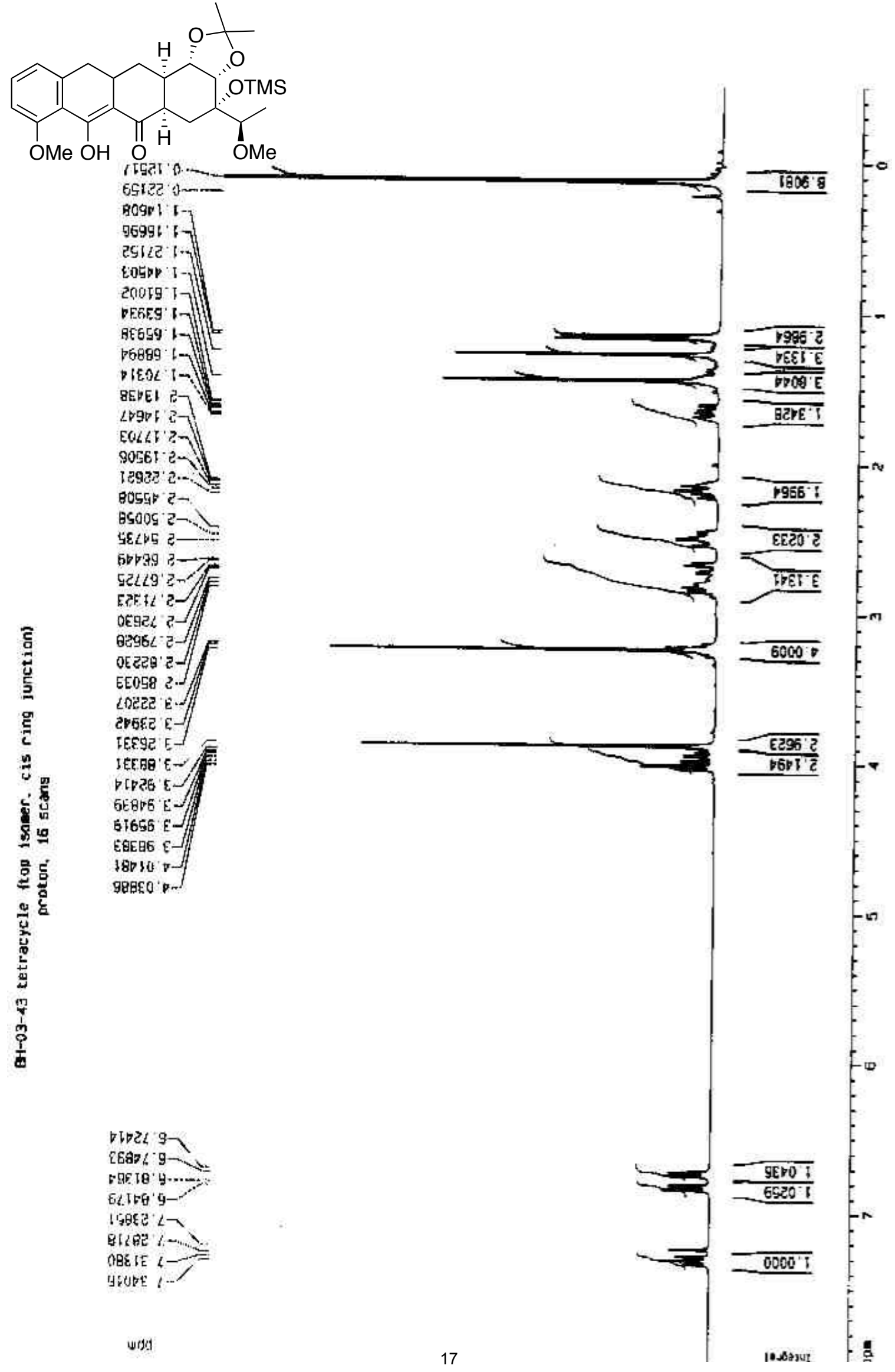




\section{Compound 7, $75 \mathrm{MHz}^{13} \mathrm{C}$ NMR $\left(\mathrm{CDCl}_{3}\right)$}

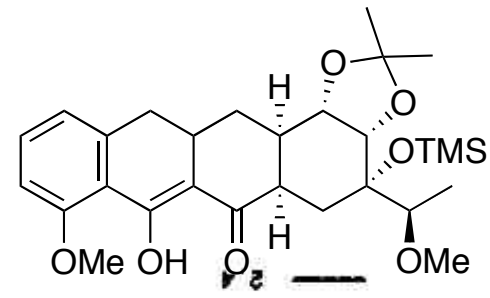
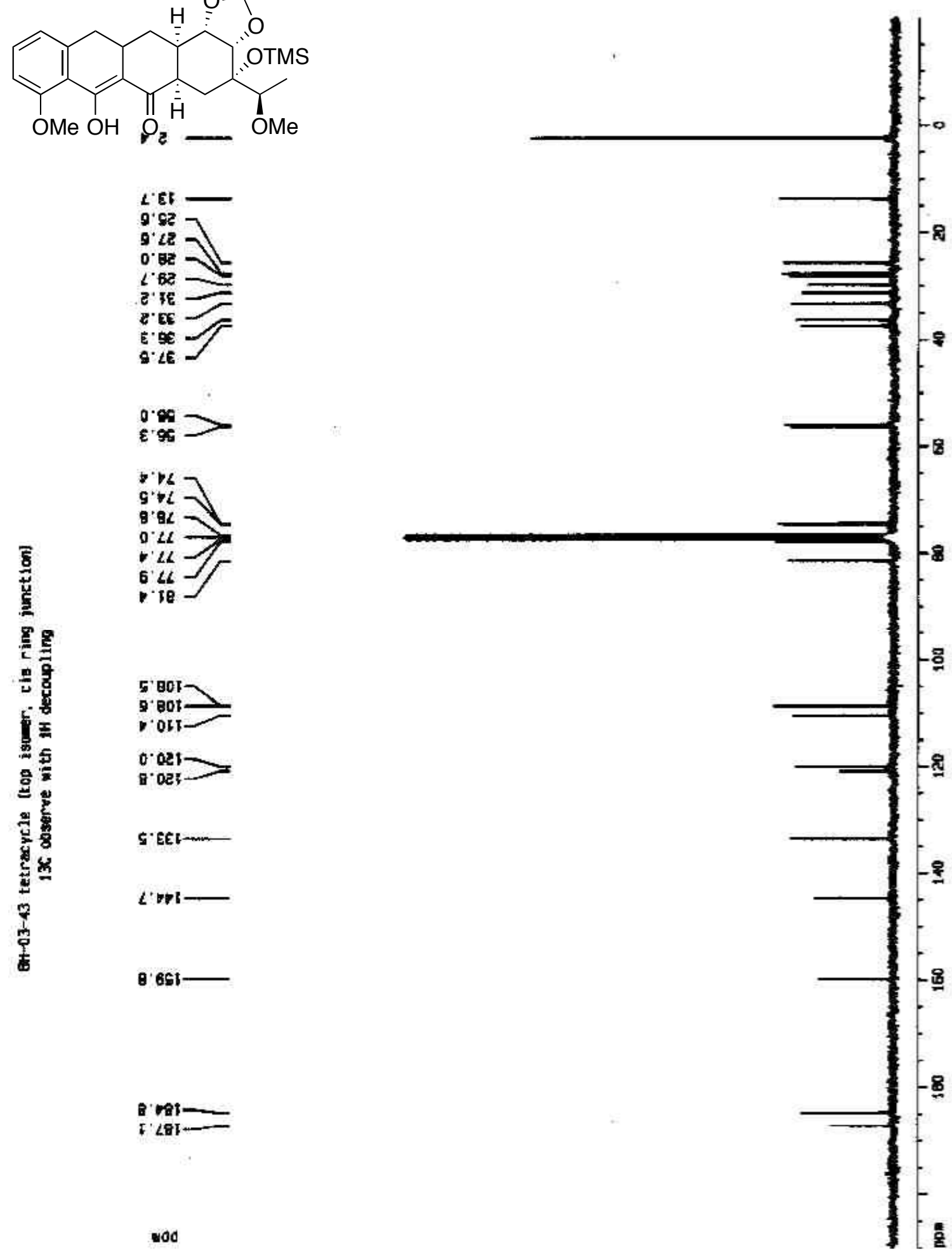
Compound 8, $300 \mathrm{MHz}^{1} \mathrm{H}$ NMR $\left(\mathrm{CDCl}_{3}\right)$<smiles>COc1cccc2c1C1=C(O)C(=O)C3(OC)OCC4C(=S)C=CCC4(C)C2C3C1</smiles>
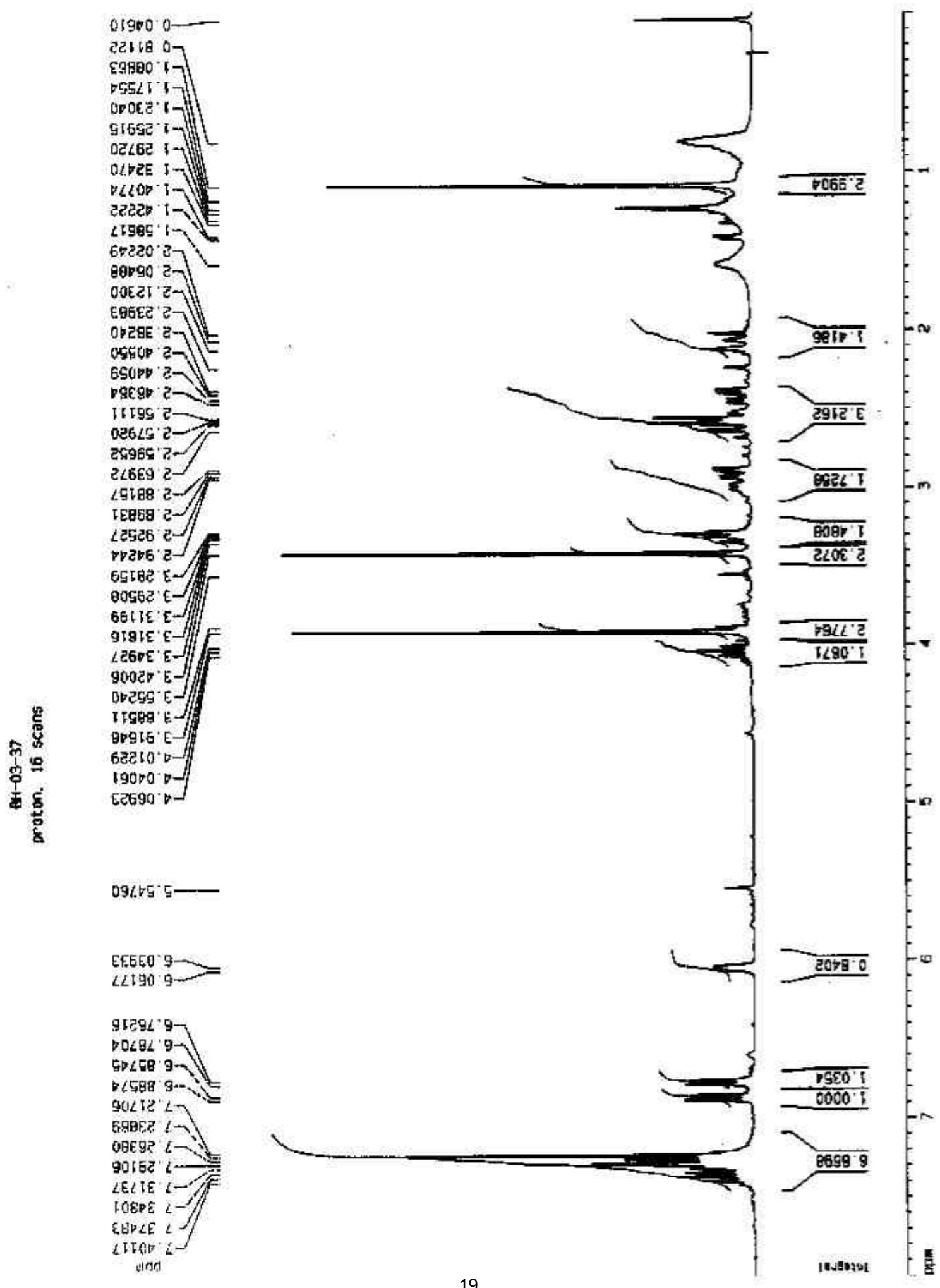